\title{
Effect of Lactic Acid Strains Isolated from Kimchi on Atopic Dermatitis and Immunomodulation in NC/Nga Mice
}

\author{
Dakyung Kim', Soo-Jeung Park', Jinkyung Kim², Unpyo Hong ${ }^{3}$, and Jeongmin Lee ${ }^{1,2}$ \\ ${ }^{1}$ Department of Medical Nutrition, Kyung Hee University, Gyeonggi 17104, Korea \\ ${ }^{2}$ Research Institute of Medical Nutrition, Kyung Hee University, Seoul 02447, Korea \\ ${ }^{3}$ Rainbowbiotech Inc., Chuncheon 24232, Korea
}

\begin{abstract}
Kimchi is a traditional Korean food, of which its constituent lactic acid bacteria have been reported to possess various physiological activities. However, few studies have investigated the immunological activity of these bacteria or their effect on atopic dermatitis (AD). We investigated whether a mixture of 6 types of lactic acid bacteria strains (LBS) isolated from kimchi has an immunomodulating effect on atopy. Mice with atopic dermatitis were orally administered LSB from kimchi for 8 weeks, and skin moisture content, scratching behavior, T-and B-cell proliferation, Th1/2 cytokines, and serum IgE and histamine levels were measured. In addition, hematoxylin and eosin and toluidine blue staining were conducted. Mice receiving LBS from kimchi had increased skin moisture content (164.3\%) and T-cell proliferation (more than 4-fold), and decreased number of scratching behaviors (78.2\%) and B-cell proliferation (63.7\%) compared with the 2,4dinitrochlorobenzene control group. In addition, LBS increased Th1 type cytokines, decreased Th2 type and pro-inflammatory cytokines, and decreased blood IgE (70.4\%), histamine (67.6\%) and mast cell levels. Therefore, it suggests that LBS of kimchi may be helpful in improving AD caused by immunological imbalance.
\end{abstract}

Keywords: atopic dermatitis, immunomodulation, kimchi, lactic acid bacteria, Th1/Th2 balance

\section{INTRODUCTION}

Transforming the gut flora by administering probiotic bacteria may be a potential approach for preventing atopic disease. A decrease in Thelper (Th) type 1 (Th1) cytokine levels may increase the prevalence of allergies (Strachan, 1989). In recent years, modification of the hygiene hypothesis has shifted away from the direct effects of Th1driven infections to an impact on the diversity of microbial exposures during gut flora formation (Yazdanbakhsh et al., 2002; Kalliomäki and Isolauri, 2003).

Atopy-related diseases are the result of a Th type 2 (Th2) biased immune responses characterized by production of Th2 cytokines such as IL-4. According to the hygiene hypothesis, if the infection-related Th1 response in neonatal and infancy is not sufficient, the Th2 response is not restricted, which may result in allergic disease. However, a Th2 reaction has been shown to exist in the absence of an allergic disease and a strong Th1 reaction (Bach, 2002; Yazdanbakhsh et al., 2002). Therefore, the role of regulatory $\mathrm{T}$ cells and induction of resistance were hypothesized as factors controlling the onset of atopic disease (Akbari et al., 2003). The gastrointestinal tract (GI) contains the largest surface of the body through which microbial products interact with the immune system. Therefore, much attention is focused on the potential for gut bacteria to play a role in the pathogenesis of atopic disease (Kalliomäki and Isolauri, 2003).

Kimchi is a mixture of traditional Korean fermented cabbage or radish with various ingredients and seasonings (Choi and Hwang, 2000). Various organic acids are produced by lactic acid bacteria during kimchi fermentation and maturation (Bang et al., 2008). Recently, many studies have been conducted to determine the functionality of lactic acid bacteria (LAB) in the fermentation process. (Chang and Chang, 2010). LAB, such as Lactobacillus spp., Weissella spp., Leuconostoc spp., and Pediococcus spp., are known to improve bowel movement and immunity, and to decrease serum immunoglobulin and cholesterol levels (Wollowski et al., 2001).

The aim of this study was to confirm whether a mixture of 6 LAB isolated from kimchi (Lactobacillus plantarum 
RBK-8, Lactobacillus brevis RBK-5, Lactobacillus pentosus RBK-3, Leuconostoc mesenteroides RBK-11, Weissella cibaria RBK-2, and Lactobacillus curvatus RBK-4) are effective against atopic immune skin disease in a NC/Nga mice with induced atopic skin disease.

\section{MATERIALS AND METHODS}

\section{Experimental animals and treatments}

Six-week-old male NC/Nga mice and age-matched Balb/c mice were purchased from Japan SLC, Inc. (Hamamatsu, Japan) and housed in wire mesh-bottomed individual cages at $24 \pm 1^{\circ} \mathrm{C}$ in $55 \pm 55 \%$ humidity with a 12 -h light/ dark cycle. All mice were acclimatized for 7 days before the experiment, fed standard pellet chow, and provided with fresh water ad libitum. A total 64 mice were randomly divided into eight groups: untreated $\mathrm{Balb} / \mathrm{c}$ mice (normal control; NC), 2,4-dinitrochlorobenzene (DNCB)-control mice $(0.1 \% \mathrm{w} / \mathrm{v}$ DNCB-treated NC/Nga mice; C), positive control mice [DNCB-treated NC/Nga mice fed a dietary supplement of $240 \mathrm{mg} / \mathrm{kg}$ of body weight (b.w.) of $\gamma$-rinolenic acid; PC], Lactobacillu alimentarius RBK-13 fed mice (DNCB-treated NC/Nga mice fed a dietary supplement of $2 \times 10^{4}$ colony-forming unit (CFU)/mouse of L. alimentarius RBK-13), L. brevis RBK-5 fed mice (DNCBtreated NC/Nga mice fed a dietary supplement of $2 \times 10^{4}$ CFU/mouse of $L$. brevis RBK-5), L. plantarum RBK-8 fed mice (DNCB-treated NC/Nga mice fed a dietary supplement of $2 \times 10^{4} \mathrm{CFU} /$ mouse of L. plantarum RBK-8), and lactic acid bacteria strains (LBS) fed mice [DNCB-treated NC/Nga mice fed a dietary supplement of $2 \times 10^{4} \mathrm{CFU} /$ mouse of LBS isolated kimchi]. Atopic dermatitis (AD)like symptoms were induced by topical application of $0.1 \% \mathrm{w} / \mathrm{v}$ DNCB (Sigma-Aldrich Co., St. Louis, MO, USA) in olive oil and acetone solution (1:3) twice a week for 2 weeks along with the diet supplement. The mice were then fed the diet supplement for a further 2 weeks without DNCB treatment. At the end of experimental periods (4 weeks), the spleens and dorsal skin tissue of all mice were dissected and blood samples were collected for use in assays. All the experiments were performed in accordance with protocols approved by the Institutional Animal Care and Use Review Committee of Kyung Hee University (KHGAST-19-268).

\section{Epidermal hydration and histopathological observation of mouse skin}

Epidermal hydration was measured with a Tewameter CM825 (Courage+Khazaka electronic GmbH, Köln, Germany) in the dorsal skin surface at the end of 5 weeks under standardized conditions $\left(22 \sim 24^{\circ} \mathrm{C}\right.$ and $55 \sim 60 \%$ humidity). Dorsal skin tissues were fixed in $10 \%$ buffered formalin, embedded in paraffin, and sliced into $5-\mu \mathrm{m}$ sections. The sections were stained with hematoxylin and eosin (H\&E) and toluidine blue for observation of mast cells by light microscopy.

\section{T- and B-cell proliferation of primary splenocytes}

First, each spleen was minced with a 40-mesh strainer in culture medium [ $10 \%$ fetal bovine serum, Roswell Park Memorial Institute (RPMI) 1640, $2 \mathrm{mM}$ glutamine, and $1 / 10^{5}$ units/L penicillin/streptomycin]. To remove any red blood cells (RBCs), cells were resuspended in RBC lysis buffer and reacted on ice for $10 \mathrm{~min}$. Cells were then washed twice with phosphate-buffered saline ( $\mathrm{pH}$ 7.4) and resuspended in complete RPMI. The splenocytes were cultured at a density of $1 \times 10^{6}$ cells/well in 96-well tissue culture dishes, and treated with concanavalin $\mathrm{A}$ (ConA; $5 \mu \mathrm{g} / \mathrm{mL}$ ) to induce T-cell proliferation or lipopolysaccharide (LPS; $5 \mu \mathrm{g} / \mathrm{mL}$ ) to induce B-cell proliferation. After $48 \mathrm{~h}$ incubation at $37^{\circ} \mathrm{C}, 10 \mu \mathrm{L}$ of EZ-CyTox (Dogen, Seoul, Korea) was added to each well, and reactions were incubated for $4 \mathrm{~h}$ at $37^{\circ} \mathrm{C}$. The absorbance at $340 \mathrm{~nm}$ was measured using an iMark ${ }^{\mathrm{TM}}$ enzyme-linked immunosorbent assay (ELISA) microplate reader (BioRad Laboratories, Hercules, CA, USA).

\section{Determination of cytokines in primary splenocytes}

Splenocytes were cultured at a density of $1 \times 10^{6}$ cells/well in 96-well tissue culture dishes and stimulated with LPS $5 \mu \mathrm{g} / \mathrm{mL}$ to induce tumor necrosis factor (TNF)- $\alpha$ and interleukin (IL)-6 production; other splenocytes were stimulated with ConA $5 \mu \mathrm{g} / \mathrm{mL}$ to induce IL-2, interferon (IFN)- $\gamma$, IL-4, and IL-10 production. After incubation for $24 \mathrm{~h}$, supernatants were collected and measured for concentrations of IL-2, IL-4, IL-10, IL-6, and TNF- $\alpha$. After incubation for $72 \mathrm{~h}$, the supernatants were collected and measured for IFN- $\gamma$ concentrations. Cytokine concentrations were analyzed using Duoset ELISA development kits (R\&D Systems, Minneapolis, MN, USA) according to the manufacturer's instructions.

\section{Determination of immunoglobulin and histamine concentrations}

Blood samples were collected from mice after sacrifice and centrifuged at 2,500 $\mathrm{g}$ for $20 \mathrm{~min}$ at $4^{\circ} \mathrm{C}$. Serum IgE levels were quantified using a mouse IgE ELISA kits (Abcam, Cambridge, MA, USA) and histamine levels were measured using mouse histamine ELISA kits (Labor Diagnostika Nord GmbH \& CoKG, Nordhorn, Germany), both according to the manufacturers' instructions.

\section{Statistical analysis}

All data were expressed as mean \pm standard deviation (SD) analyzed by the one-way ANOVA using SPSS statistical software for Windows (SPSS PASW 23.0; SPSS Inc., Chicago, IL, USA). Duncan's multiple range post-hoc tests 
were used for examining differences between groups. A $P$ value of $<0.05$ was considered statistically significant.

\section{RESULTS}

Effects of LBS on weight gain, food consumption, food efficiency rate (FER), and spleen weight

Weight gain, food consumption, FER, and spleen weight of the mice are shown in Table 1. There were no significant differences in body weight or food consumption between the groups. However, in all DBNC groups, FER was significantly decreased, and spleen weight was significantly increased compared the $\mathrm{C}$ group.

\section{Effects of LBS on scratching behavior of DNCB-treated $\mathrm{NC} /$ Nga mice}

Scratching behavior was significantly increased in the C group compared to the NC group. Furthermore, scratching behavior of the $\mathrm{PC}$ and $L$. alimentarius $\mathrm{RBK}-13$ groups was significantly decreased compared to the $\mathrm{C}$ group, but there was no significant difference between $L$. brevis RBK-5 and L. plantarum RBK-8 groups compared with the C group. However, scratching behavior was significantly lower in the LBS group compared with the $\mathrm{C}$ group $(P<$ 0.05) (Fig. 1A).

\section{Effects of LBS on epidermal hydration in DNCB-treated $\mathrm{NC} /$ Nga mice}

Epidermal hydration was significantly decreased in the $\mathrm{C}$ group compared with the NC group. In addition, epidermal hydration in the PC, L. alimentarius RBK-13, L. brevis RBK-5, and plantarum RBK-8 groups was significantly elevated compared with the $C$ group. Specifically, epidermal hydration was significantly elevated in the LBS group compared with the $\mathrm{C}$ group $(P<0.05)$ (Fig. 1B).

Table 1. Body weight gain, food efficiency ratio, and spleen weight of experimental animals for 8 weeks

\begin{tabular}{|c|c|c|c|c|c|c|c|}
\hline \multirow[b]{2}{*}{ Measurements } & \multirow[b]{2}{*}{ NC } & \multicolumn{6}{|c|}{ DNCB } \\
\hline & & $\mathrm{C}$ & $P C$ & $\begin{array}{l}\text { L. alimentarius } \\
\text { RBK-13 }\end{array}$ & $\begin{array}{l}\text { L. brevis } \\
\text { RBK-5 }\end{array}$ & $\begin{array}{l}\text { L. plantarum } \\
\text { RBK-8 }\end{array}$ & $\begin{array}{l}\text { LSB from } \\
\text { kimchi }\end{array}$ \\
\hline Weight gain $(g)^{1)}$ & $10.70 \pm 1.18^{\mathrm{ns}}$ & $10.48 \pm 0.82$ & $10.37 \pm 1.57$ & $10.33 \pm 0.68$ & $10.28 \pm 0.47$ & $10.42 \pm 1.20$ & $10.27 \pm 1.05$ \\
\hline Food consumption $(\mathrm{g} / \mathrm{d})$ & $2.90 \pm 0.62^{\mathrm{ns}}$ & $3.55 \pm 0.88$ & $3.38 \pm 0.93$ & $3.28 \pm 0.88$ & $3.27 \pm 0.97$ & $3.33 \pm 0.86$ & $3.33 \pm 0.90$ \\
\hline FER $^{2)}$ & $5.27 \pm 0.58^{\mathrm{a}}$ & $3.95 \pm 0.43^{b}$ & $3.43 \pm 0.63^{b}$ & $3.77 \pm 0.38^{b}$ & $3.47 \pm 0.31^{b}$ & $4.11 \pm 0.63^{b}$ & $3.69 \pm 0.26^{b}$ \\
\hline Spleen (g) & $0.23 \pm 0.03^{b}$ & $0.54 \pm 0.19^{a}$ & $0.57 \pm 0.19^{a}$ & $0.43 \pm 0.06^{\mathrm{a}}$ & $0.48 \pm 0.08^{a}$ & $0.56 \pm 0.08^{\mathrm{a}}$ & $1.04 \pm 0.96^{\mathrm{a}}$ \\
\hline
\end{tabular}

Results are expressed as mean \pm SD.

Means with different letters $(a, b)$ are significantly different at $P<0.05$.

NC, normal control; C, DNCB-control; PC, positive control (DNCB-treated NC/Nga mice fed a disupplement of $240 \mathrm{mg} / \mathrm{kg}$ b.w. of $\gamma$-rinolenic acid); lactic acid bacteria strains (LBS) from kimchi [DNCB-treated NC/Nga mice fed a dietary supplement of $2 \times 10^{4}$ CFU/mouse of LBS isolated kimchi].

${ }^{1)}$ Weight gain $(\mathrm{g} / 2$ weeks)=final body weight $(\mathrm{g})$-initial body weight $(\mathrm{g})$.

${ }^{2)}$ Food efficiency ratio=weight gain $(\mathrm{g}) /$ total food consumption $(\mathrm{g}) \times 100$.

A

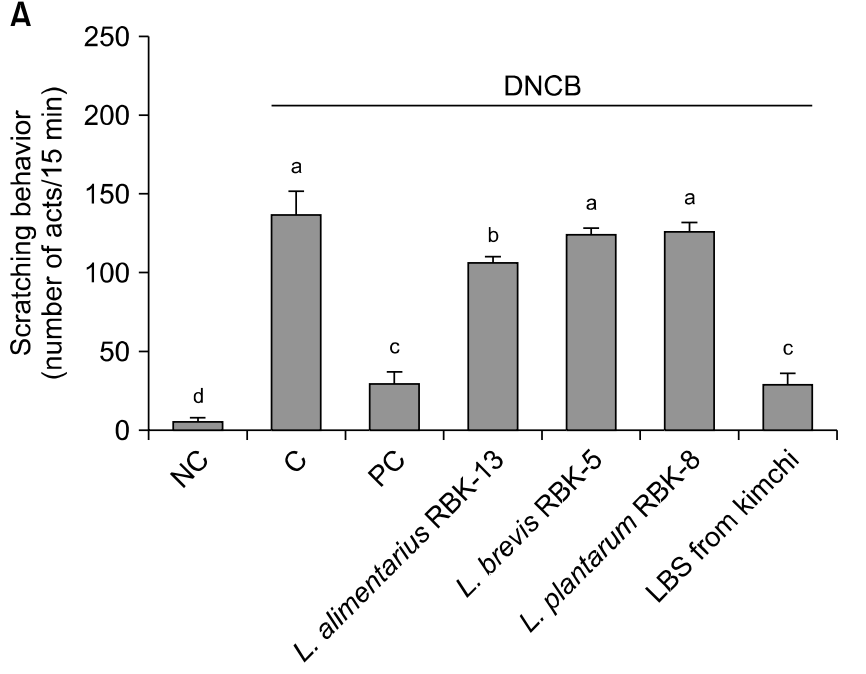

B

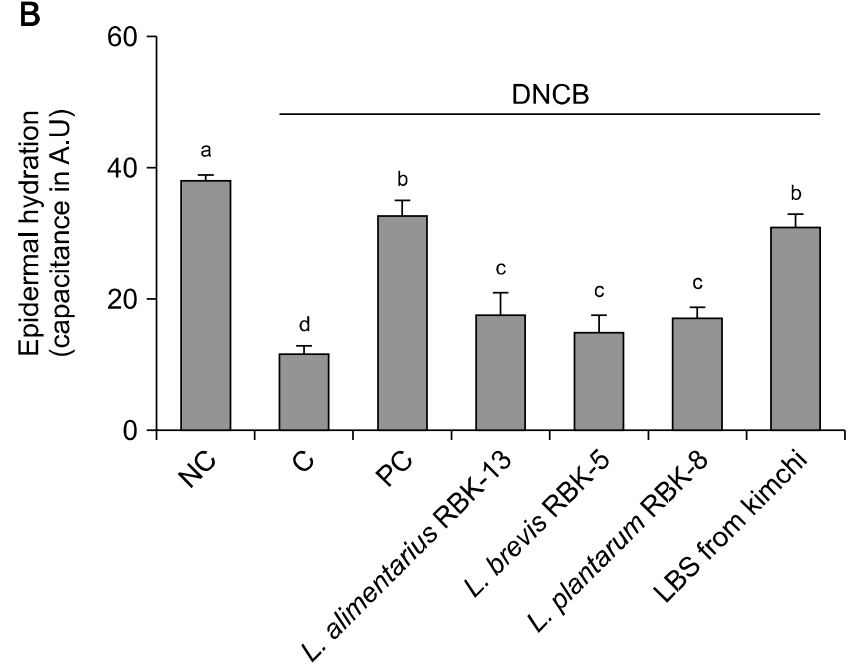

Fig. 1. Change of skin scratching behavior (A) and epidermal hydration of skin (B) in mice. Results are expressed as mean $\pm \mathrm{SD}$. Means with different letters (a-d) are significantly different at $P<0.05$. NC, normal control; $\mathrm{C}$, DNCB-control; PC, positive control (DNCB-treated NC/Nga mice fed a di-etary supplement of $240 \mathrm{mg} / \mathrm{kg}$ b.w. of $\gamma$-rinolenic acid); lactic acid bacteria stains (LBS) from kimchi [DNCB-treated NC/Nga mice fed a dietary supplement of $2 \times 10^{4} \mathrm{CFU} /$ mouse of LBS isolated kimchi]. 
Effects of LBS on T- and B-cell proliferation in primary splenocytes from DNCB-treated NC/Nga mice

T-cell proliferation of mitogen (ConA)-stimulated splenocytes was significantly lower in the $\mathrm{C}$ group compared with the NC group. Furthermore, T-cell proliferation in the PC, L. alimentarius RBK-13, L. brevis RBK-5, and plantarum RBK-8 groups was significantly increased compared to the $\mathrm{C}$ group. Specifically, the LBS group showed the most significant difference compared with the $\mathrm{C}$ group $(P<0.05)$ (Fig. $2 \mathrm{~A})$.

B-cell proliferation of mitogen (LPS)-stimulated splenocytes was significantly greater in the $\mathrm{C}$ group compared with the NC group. However, B-cell proliferation in the
$\mathrm{PC}$, L. alimentarius RBK-13, L. brevis RBK-5, and L. plantarum RBK-8 groups was significantly lower compared with the C group. Specifically, the LBS group showed the greatest significant difference compared with the $\mathrm{C}$ group. $(P<0.05)$ (Fig. 2B)

\section{Effects of LBS on Th1-type cytokines in primary spleno- cytes of DNCB-treated NC/Nga mice}

Th1-type cytokine (IL-2 and IFN- $\gamma$ ) production in primary splenocytes is shown in Fig. 3. IL-2 and IFN- $\gamma$ production from ConA-stimulated splenocytes were significantly lower in the $\mathrm{C}$ group compared with the $\mathrm{NC}$ group. In addition, productions of IL- 2 and IFN- $\gamma$ in the PC, L. ali-
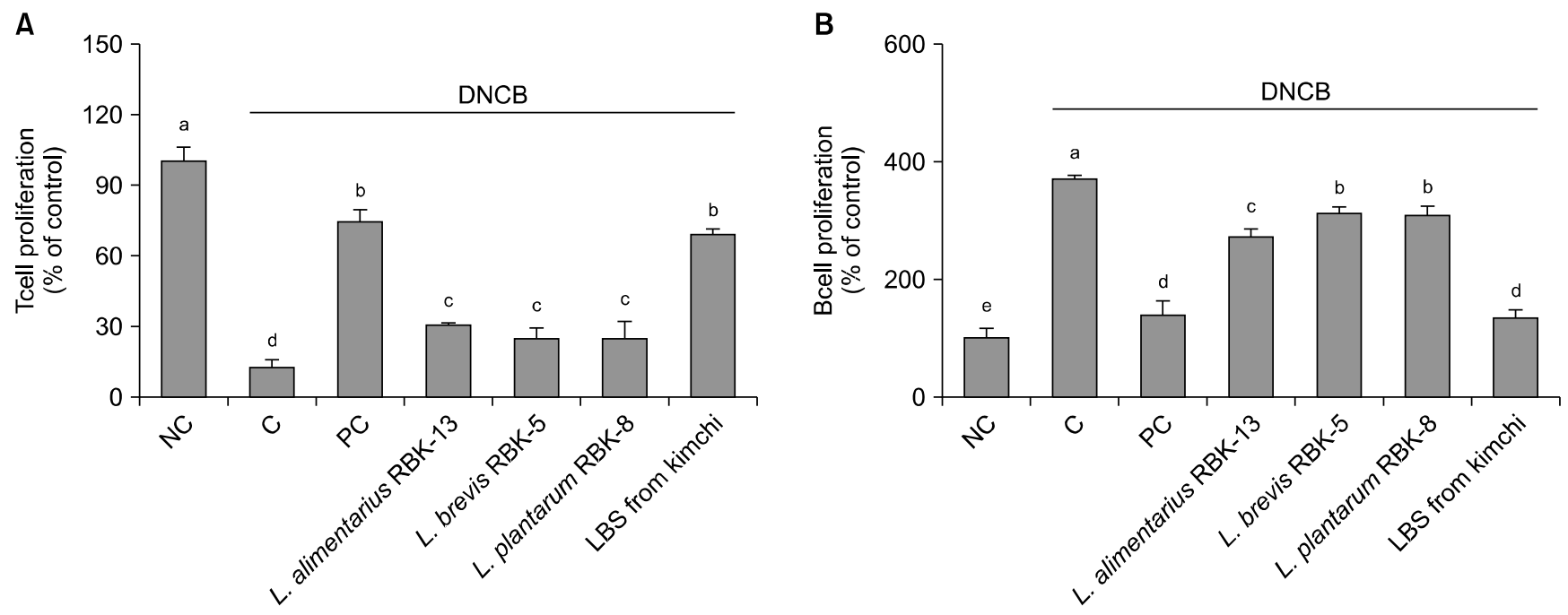

Fig. 2. Effect of Lactobacillus bacteria and lactic acid bacteria mixture on T-cell (A) and B-cell (B) proliferation from primary splenocytes. Results are expressed in mean \pm SD. Means with different letters (a-e) are significantly different at $P<0.05$. NC, normal control; C, DNCB-control; PC, positive control (DNCB-treated NC/Nga mice fed a dietary supplement of $240 \mathrm{mg} / \mathrm{kg}$ b.w. of $\gamma$-rinolenic acid); lactic acid bacteria stains (LBS) from kimchi [DNCB-treated NC/Nga mice fed a dietary supplement of $2 \times 10^{4} \mathrm{CFU} / \mathrm{mouse}$ of LBS isolated kimchi].
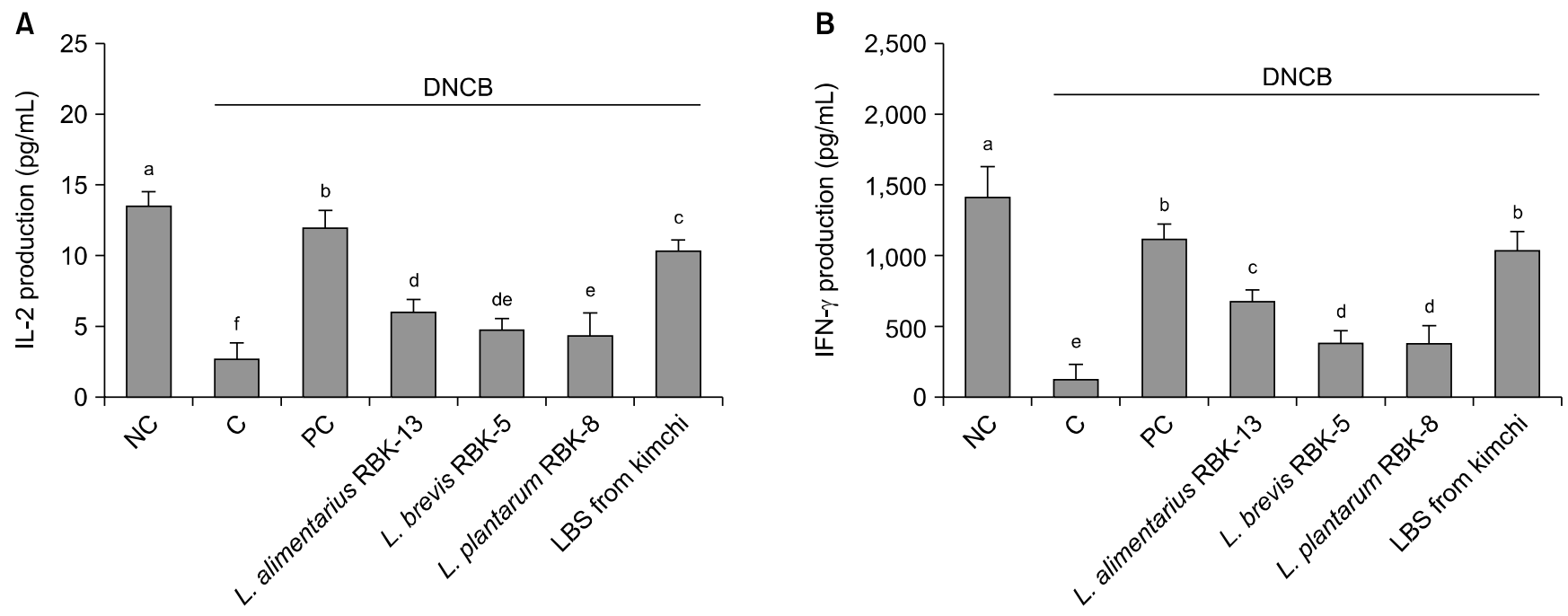

Fig. 3. Effect of Lactobacillus bacteria and lactic acid bacteria mixture on Th1 type cytokine (A: IL-2 and B: IFN- $\gamma$ ) production from ConA stimulated primary splenocytes. Results are expressed as mean $\pm S D$. Means with different letters (a-f) are significantly different at $P<0.05$. NC, normal control; C, DNCB-control; PC, positive control (DNCB-treated NC/Nga mice fed a dietary supplement of $240 \mathrm{mg} / \mathrm{kg}$ b.w. of $\gamma$-rinolenic acid); lactic acid bacteria stains (LBS) from kimchi [DNCB-treated NC/Nga mice fed a dietary supplement of $2 \times 10^{4} \mathrm{CFU} /$ mouse of LBS isolated kimchi]. 
mentarius RBK-13, L. brevis RBK-5, and L. plantarum RBK8 groups were significantly higher compared with the C group. Specifically, IL-2 and IFN- $\gamma$ productions were significantly higher in the LBS group compared with the $\mathrm{C}$ group $(P<0.05)$.

\section{Effects of LBS on Th2-type cytokines in primary spleno- cytes of DNCB-treated NC/Nga mice}

Th2-type cytokine (IL-4 and IL-10) production in primary splenocytes is shown in Fig. 4. IL-4 and IL-10 production from ConA-stimulated splenocytes were significantly higher in the $\mathrm{C}$ group compared with the $\mathrm{NC}$ group. In addition, production of IL-4 and IL-10 was significantly lower in the PC, L. alimentarius RBK-13, L. brevis RBK-5, and plantarum RBK-8 groups compared with the $\mathrm{C}$ group. Specifically, IL-4 and IL-10 productions were significantly lower in the LBS group compared with the C group $(P<0.05)$.

\section{Effects of LBS on pro-inflammatory cytokines in primary splenocytes of DNCB-treated NC/Nga mice} Th2-type cytokine (IL- 6 and TNF- $\alpha$ ) production in primary splenocytes is shown in Fig. 5. IL-6 and TNF- $\alpha$ production from LPS-stimulated splenocytes were significantly higher in the $\mathrm{C}$ group compared with the $\mathrm{NC}$ group. In addition, IL- 6 and TNF- $\alpha$ productions in the PC, $L$.
A

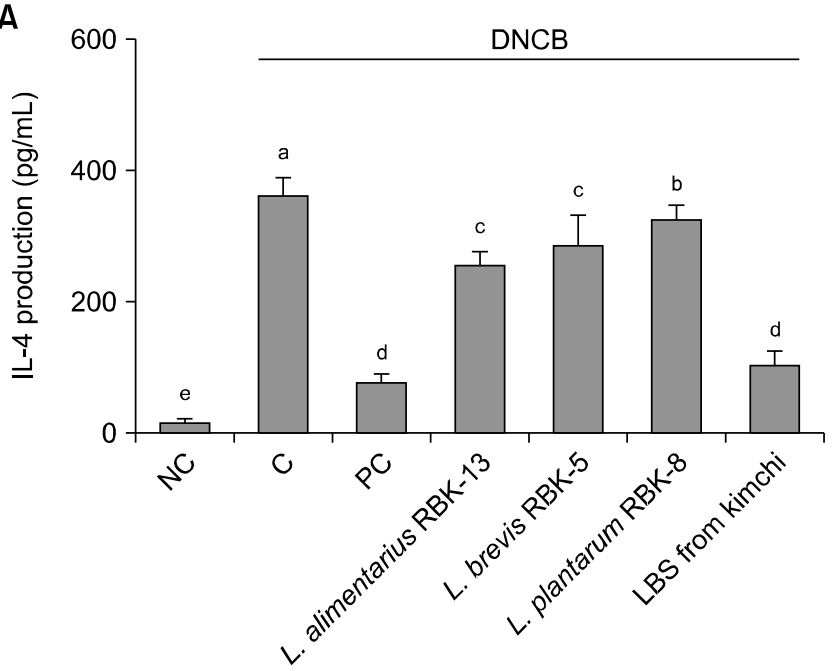

B

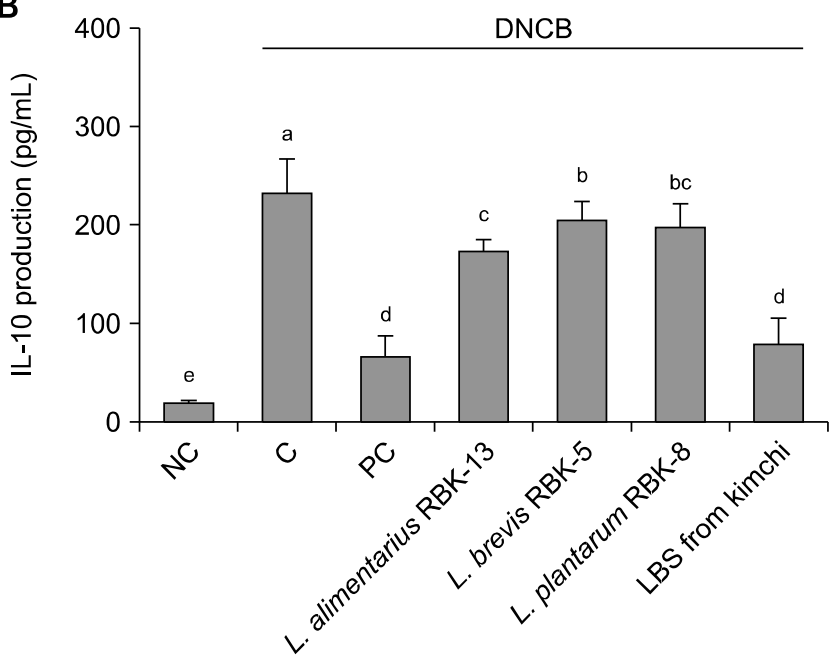

Fig. 4. Effect of Lactobacillus bacteria and lactic acid bacteria mixture on Th2 type cytokine (A: IL-4 and B: IL-10) production from Con-A stimulated primary splenocytes. Results are expressed as mean \pm SD. Means with different letters (a-e) are significantly different at $P<0.05$. NC, normal control; C, DNCB-control; PC, positive control (DNCB-treated NC/Nga mice fed a dietary supplement of $240 \mathrm{mg} / \mathrm{kg}$ b.w. of $\gamma$-rinolenic acid); lactic acid bacteria stains (LBS) from kimchi [DNCB-treated NC/Nga mice fed a dietary supplement of $2 \times 10^{4} \mathrm{CFU} /$ mouse of LBS isolated kimchi].

A

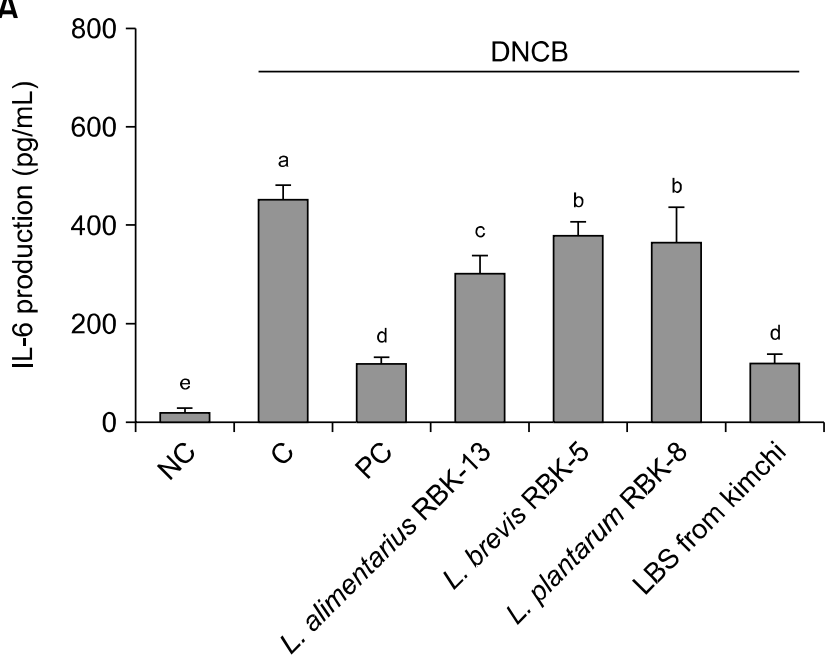

B

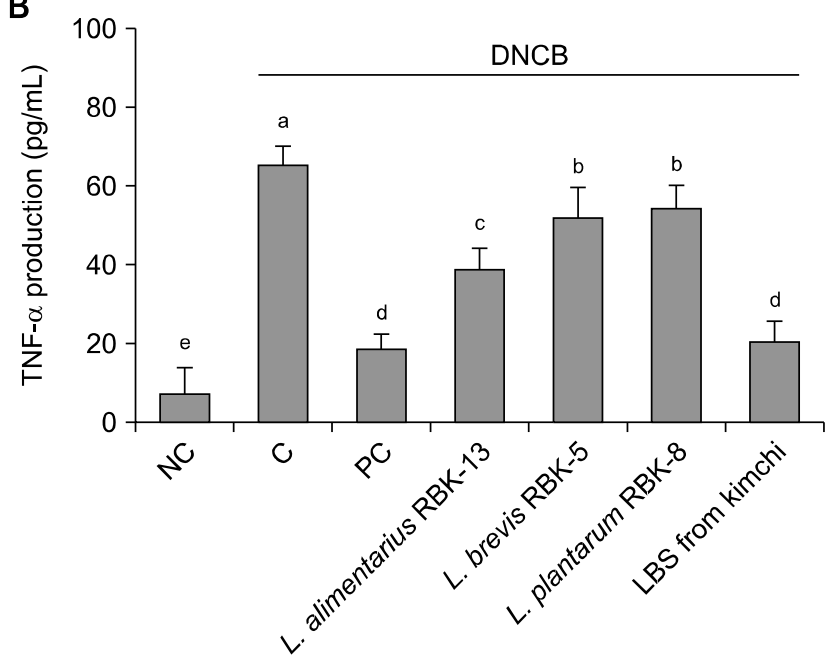

Fig. 5. Effect of Lactobacillus bacteria and lactic acid bacteria mixture on IL-6 (A) and TNF- $\alpha$ (B) production from LPS-stimulated primary splenocytes. Results are expressed as mean \pm SD. Means with different letters (a-e) are significantly different at $P<0.05$. NC, normal control; C, DNCB-control; PC, positive control (DNCB-treated NC/Nga mice fed a dietary supplement of $240 \mathrm{mg} / \mathrm{kg}$ b.w. of $\gamma$-rinolenic acid); lactic acid bacteria stains (LBS) from kimchi [DNCB-treated NC/Nga mice fed a dietary supplement of $2 \times 10^{4} \mathrm{CFU} /$ mouse of LBS isolated kimchi]. 
alimentarius RBK-13, L. brevis RBK- 5 , and L. plantarum RBK-8 groups were significantly lower compared with the C group. Specifically, IL- 6 and TNF- $\alpha$ productions were significantly lower in the LBS group compared with the C group $(P<0.05)$.

\section{Effects of IgE concentrations in the serum of DNCB-treated $\mathrm{NC} /$ Nga mice}

Serum IgE concentrations were significantly higher in the $\mathrm{C}$ group than the $\mathrm{NC}$ group. In addition, serum IgE concentrations in the $\mathrm{PC}$ and $L$. alimentarius RBK-13 groups were significantly lower than those of the $\mathrm{C}$ group. There were no significant differences between the $L$. brevis RBK5, L. plantarum RBK-8 and C groups. However, IgE levels in the LBS group were significantly lower than those of the $\mathrm{C}$ group $(P<0.05)$ (Fig. $6 \mathrm{~A})$.

\section{Effects of histamine concentrations in the serum of DNCB- treated NC/Nga mice}

Serum histamine concentrations were significantly higher in the $\mathrm{C}$ group than the $\mathrm{NC}$ group. In addition, serum histamine concentrations in the PC, L. alimentarius RBK-
A

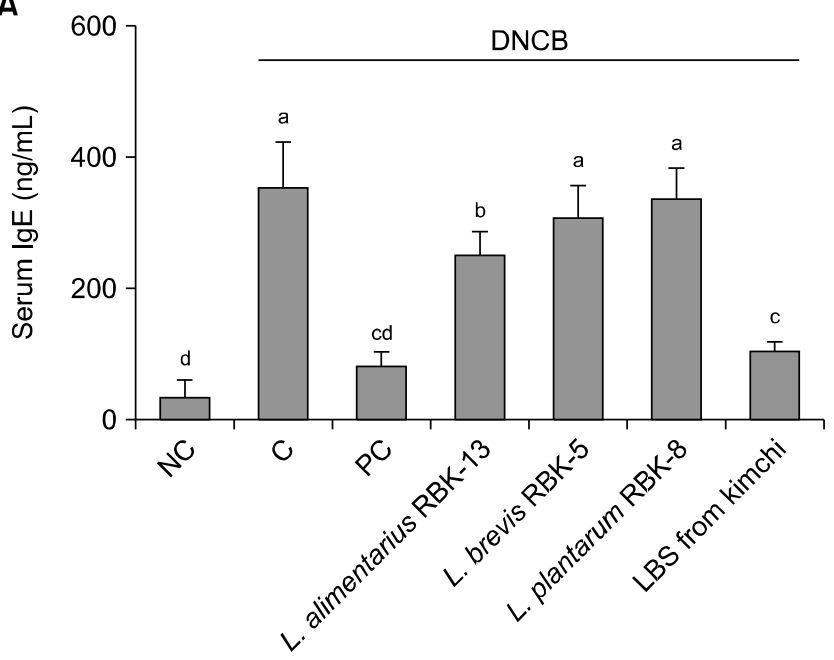

B

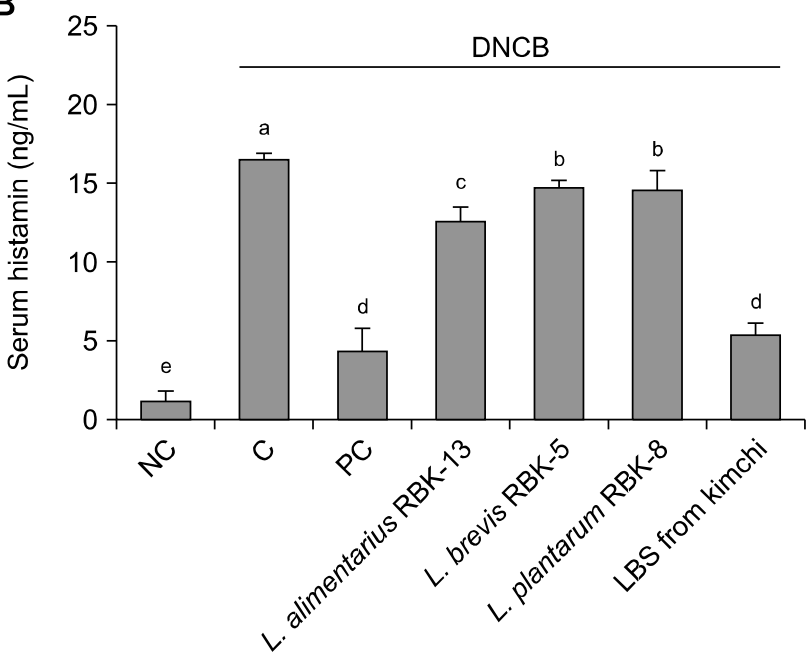

Fig. 6. Effect of Lactobacillus bacteria and lactic acid bacteria mixture on serum IgE (A) and histamine (B) production in mice. Results are expressed as mean \pm SD. Means with different letters (a-e) are significantly different at $P<0.05$. NC, normal control; C, DNCB-control; PC, positive control (DNCB-treated NC/Nga mice fed a dietary supplement of $240 \mathrm{mg} / \mathrm{kg} \mathrm{b.w}$. of $\gamma$-rinolenic acid); lactic acid bacteria stains (LBS) from kimchi [DNCB-treated NC/Nga mice fed a dietary supplement of $2 \times 10^{4}$ CFU/mouse of LBS isolated kimchi].

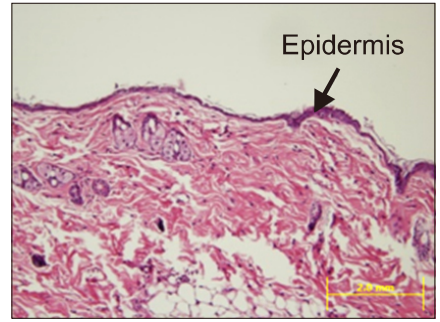

NC

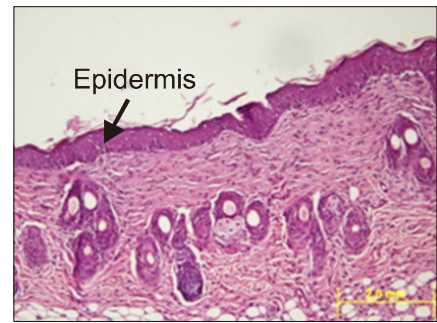

Lactobacillus brevis RBK-5
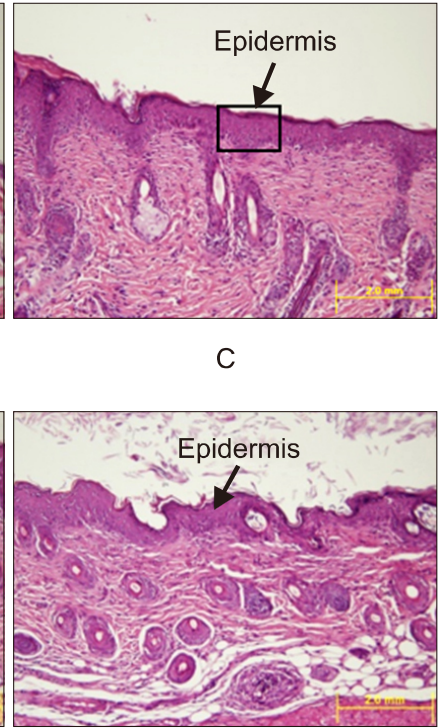

Lactobacillus plantarum RBK-8

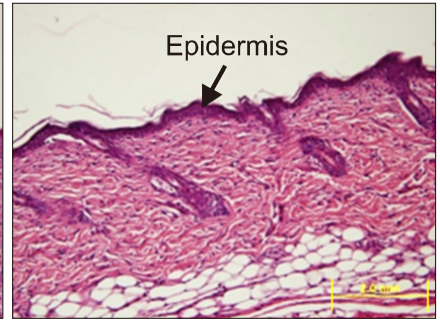

$\mathrm{PC}$

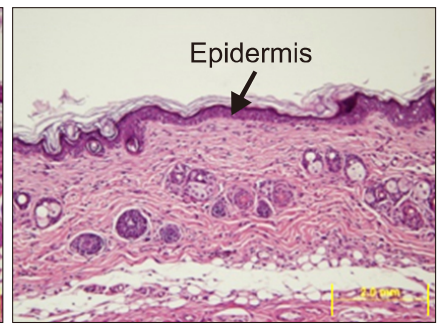

LBS from kimchi

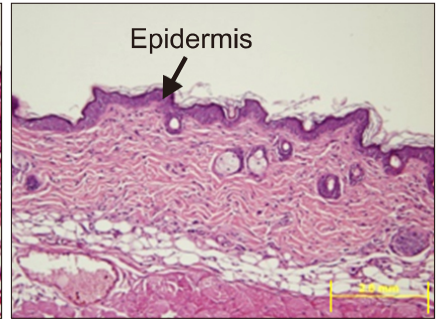

Lactobacillus alimentarius RBK-13

Fig. 7. Effect of Lactobacillus bacteria and lactic acid bacteria mixture on epidermis thickness of the experimental mice. NC, normal control; C, DNCB-control; PC, positive control (DNCB-treated NC/Nga mice fed a dietary supplement of $240 \mathrm{mg} / \mathrm{kg}$ b.w. of $\gamma$-rinolenic acid); lactic acid bacteria stains (LBS) from kimchi [DNCB-treated NC/Nga mice fed a dietary supplement of $2 \times 10^{4}$ CFU/mouse of LBS isolated kimchi]. 
A

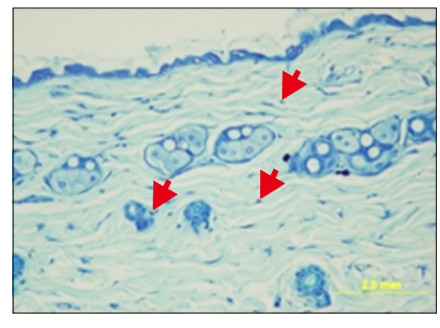

NC

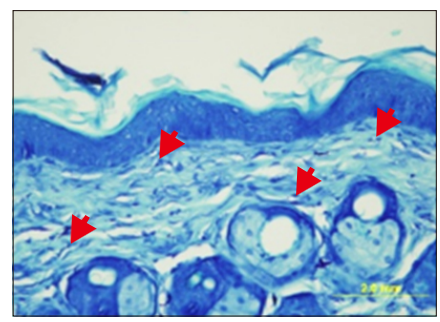

Lactobacillus brevis RBK-5

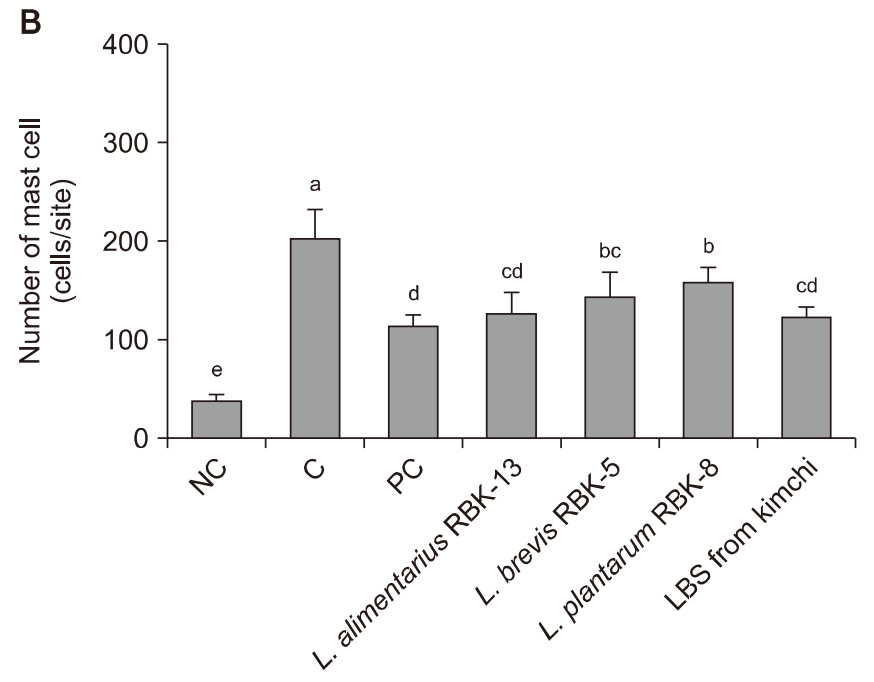

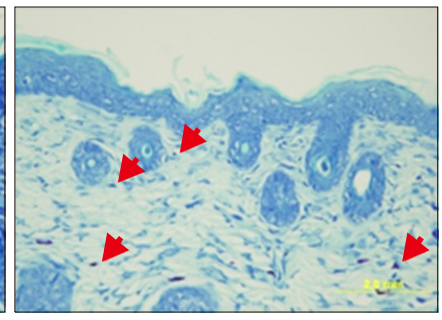

PC

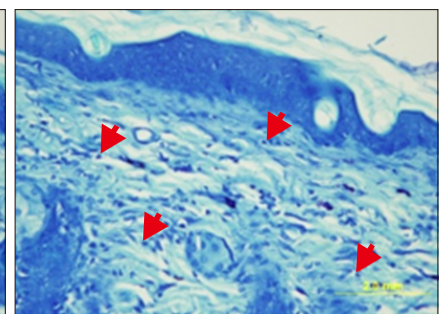

Lactobacillus

plantarum RBK-8

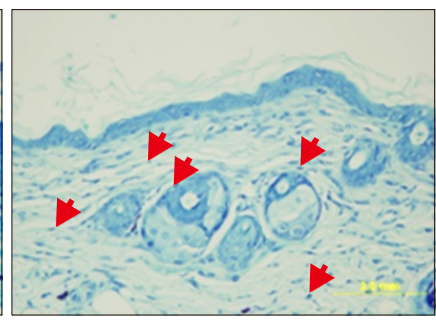

LBS from kimchi

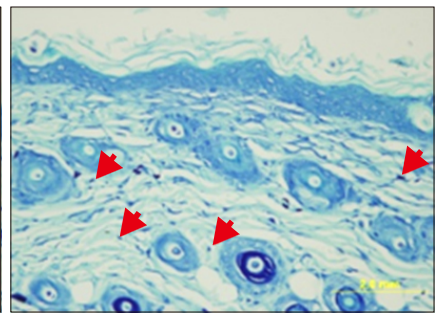

Lactobacillus

alimentarius RBK-13

Fig. 8. Effect of Lactobacillus bacteria and lactic acid bacteria mixture on Toluidin blue staining and the number of mast cells of the experimental mice. Results are expressed as mean $\pm S D$. Means with different letters (a-e) are significantly different at $P<0.05$. NC, normal control; $\mathrm{C}$, DNCB-control; $\mathrm{PC}$, positive control (DNCB-treated $\mathrm{NC} / \mathrm{Nga}$ mice fed a dietary supplement of $240 \mathrm{mg} / \mathrm{kg}$ b.w. of $\gamma$-rinolenic acid); lactic acid bacteria stains (LBS) from kimchi [DNCB-treated NC/Nga mice fed a dietary supplement of $2 \times 10^{4}$ CFU/mouse of LBS isolated kimchi].

13, L. brevis RBK-5, and L. plantarum RBK-8 groups were significantly lower than those of the $\mathrm{C}$ group. Specifically, histamine level in the LBS group were significantly lower than those of the $\mathrm{C}$ group $(P<0.05)$ (Fig. $6 \mathrm{~B})$.

\section{Effects of LBS on epidermis thickness and mast cell counts of DNCB-treated NC/Nga mice}

Epidermal thickness was significantly higher in the $\mathrm{C}$ group than the NC group. In addition, epidermal thicknesses in the PC, L. alimentarius RBK-13, L. brevis RBK-5, and $L$. plantarum RBK-8 groups were significantly lower compared with the $\mathrm{C}$ group. Furthermore, epidermal thickness was significantly lower in the LBS group compared with the $\mathrm{C}$ group $(P<0.05)$ (Fig. 7).

The number of mast cells was significantly higher in the $\mathrm{C}$ group compared with the $\mathrm{NC}$ group. However, mast cell counts in the PC, L. alimentarius RBK-13, L. brevis RBK-5, and L. plantarum RBK-8 groups were significantly lower compared with the $\mathrm{C}$ group. Furthermore, mast cell count was significantly lower in the LBS group compared with the $\mathrm{C}$ group $(P<0.05)$ (Fig. 8).

\section{DISCUSSION}

In this study, we showed that a mixture of six LBS isolated from kimchi reduced $\mathrm{AD}$ responses by modulating immune responses in DNCB-treated NC/Nga mice. NC/ Nga mice spontaneously develop AD-like skin lesions in response to environmental factors under conventional laboratory conditions without air filtration. NC/Nga mice with $\mathrm{AD}$ pathogenesis are characterized by morphological symptoms such as erythema, itching, hemorrhage, hyperkeratosis, and xerosis, and have several similarities to patients with AD (Suto et al., 1999). We showed that erythema, hemorrhage, and hyperkeratosis occurred on the dorsal skin of NC/Nga mice. Furthermore, that NC/ Nga mice showed decreased epidermal hydration and in- 
creased frequency of scratching compared with untreated $\mathrm{Balb} / \mathrm{c}$ mice. Increased skin hydration, which plays an important role in maintaining the skin's moisture balance, can improve $\mathrm{AD}$, whereas disrupting the balance with increased skin dryness can worsen $\mathrm{AD}$ (Darlenski et al., 2013). In addition, the NC/Nga mice had significantly heavier spleens compared with untreated $\mathrm{Balb} / \mathrm{c}$ mice $(P<0.05)$. Enlargement of the spleen may be caused by infectious conditions and diseases (Iida et al., 2000).

Our results indicate that the DNCB-treated NC/Nga mice used in this study developed a typical $\mathrm{AD}$ pathogenesis. However, administration of LBS isolated from kimchi attenuated macroscopic and histopathological changes and alleviated the reduced hydration in the dorsal skin of NC/Nga mice compared with DNCB-treated control mice. In particular, LBS induced a significant decrease in the number of scratching episodes of NC/Nga mice compared with control mice.

Pro-inflammatory cytokines induce recruitment of pathogenic white blood cells and support lymphocyte proliferation in the early stages of skin disease (van der Aa et al., 2012). Homey et al. (2006) reported that chemokines and pro-inflammatory cytokines may play a role in regulating innate and adaptive immunity at sites of atopic skin injury, leading to development of AD.

CD4+ T-helper cells are divided into Th1 and Th2 cells according to the characteristics of cytokine production. Th1 cells produce IL- 2 and IFN- $\gamma$ (Th1 type cytokine), whereas Th2 cells produce IL-4 and IL-10 (Th2 type cytokine) (Zhang et al., 1995). Th1-type cytokines are responsible for macrophage activation, cytotoxic T-cell development, and delayed-type hypersensitivity, whereas Th2-type cytokines are responsible for B-cell responses and antibody development (Zhang et al., 1995; Werfel and Wittmann, 2008). In the beginning stages of $A D$, Th2-type cytokines play an important role in pathogenesis (Werfel and Wittmann, 2008). An increase in IL-4, one of the Th2-type cytokines in the blood, is associated with $\mathrm{AD}$, as shown by its correlation with serum $\mathrm{IgE}$ and IgG1 levels that activate mast cells (Carmi-Levy et al, 2011; Grewe et al., 1998).

Mast cells contain high affinity receptors for the Fc region of IgE. When IgE binds to mast cells, it releases histamine, triggering an allergic reaction such as causing the surrounding skin to become itchy (a characteristic of AD) (Zhang et al., 1995; Matsuda et al., 1997; Werfel and Wittmann, 2008). It has previously been reported that in the spleen of NC/Nga mice serum IgE levels are significantly elevated, and IL-4 is released by CD4+ T helper cells (Matsuda et al., 1997). Data from the present study showed a marked increase in the production of serum IgE, histamine, and Th2-type cytokines in the DNCB control mice compared with $\mathrm{NC}$ mice. However, in the $\mathrm{AD}$ control group, Th1-type cytokine production by splenocytes was significantly reduced compared with the NC group $(P<0.05)$. In addition, in the DNCB group, T-cell proliferation was decreased, and B-cell proliferation was increased compared with the NC group.

This coincided with reduced production of Th2 cytokines, but substantial induction of IL-2 and IFN- $\gamma$. Based on our T-type activation tests, we speculated that the effects of probiotics will be more pronounced in antigendriven stimulation since the antigen is presented in close contact with $\mathrm{T}$ cells and antigen presenting cells.

Atopic disease is characterized by a dominant Th2 response, including IL-4 and IL-10. Our results show that LBS from kimchi may have a beneficial effect on atopic diseases by reducing production of Th2 cytokines (Murosaki et al., 1998; Shida et al, 1998; Pochard et al., 2002). In a previous study (Pochard et al., 2002), downregulation of IL-4 and IgE production by pro-inflammatory cytokines (such as IFN- $\gamma$ or IL-12) inhibited the Th2 response. However, our study suggests that the Th2 inhibitory effect of probiotics may be mediated by immunomodulatory functions that are not associated with IL-2 or IFN- $\gamma$ secretion, but may be mediated by induction of regulatory cytokines such as IL-10.

Recent studies have reported the importance of IL-10 in the regulation of immune responses by probiotic bacteria. In addition, in mouse models, certain probiotics improved colitis by inducing IL-10 production from native layer mononuclear cells (Di Giacinto et al., 2005). Production of cytokines from CD4+ T-helper cells and skin-infiltrating immune cells may play a key role in regulating pathological skin responses associated with $\mathrm{AD}$. Therefore, the balance of Th1-/Th2-type cytokines plays a crucial role in immunomodulation and AD improvement.

In the present study, oral administration of LBS isolated from kimchi attenuated Th1-/Th2- type cytokine imbalances, and production of serum IgE and histamine. The results suggest that LBS can attenuate activation and infiltration of mast cells in the skin by inhibiting IgE production in B cells induced by Th2-type cytokines.

While many studies have been conducted to confirm the function of LAB during fermentation, LAB such as Lactobacillus spp., Weissella spp., Leuconostoc spp., and Pediococcus spp. are known to be effective in defecation, improving immunity, and reducing serum and cholesterol levels (Wollowski et al., 2001).

In conclusion, this study demonstrated that LBS isolated from kimchi improves immune responses in NC/ Nga mice, including increases in IgE and histamine for $\mathrm{AD}$ through attenuating Th1/Th2 cytokine imbalances, and production of proinflammatory cytokines. LBS isolated from kimchi may be beneficial for improving AD. 


\section{ACKNOWLEDGEMENTS}

This study was supported by $R \& D$ project which conducted by the Korea Industrial Complex Corporation (KICOX).

\section{AUTHOR DISCLOSURE STATEMENT}

The authors declare no conflict of interest.

\section{REFERENCES}

Akbari O, Stock P, DeKruyff RH, Umetsu DT. Role of regulatory $\mathrm{T}$ cells in allergy and asthma. Curr Opin Immunol. 2003. 15: 627-633.

Bach JF. The effect of infections on susceptibility to autoimmune and allergic diseases. N Engl J Med. 2002. 347:911-920.

Bang BH, Seo JS, Jeong EJ. A method for maintaining good kimchi quality during fermentation. Korean J Food Nutr. 2008. 21:5155.

Carmi-Levy I, Homey B, Soumelis V. A modular view of cytokine networks in atopic dermatitis. Clin Rev Allergy Immunol. 2011. 41:245-253.

Chang JY, Chang HC. Improvements in the quality and shelf life of kimchi by fermentation with the induced bacteriocin-producing strain, Leuconostoc citreum GJ7 as a starter. J Food Sci. 2010. 75:M103-M110.

Choi HS, Hwang JH. Antioxidative characteristics of kimchi. Food Industry and Nutrition. 2000. 5(3):52-56.

Darlenski R, Kazandjieva J, Tsankov N, Fluhr JW. Acute irritant threshold correlates with barrier function, skin hydration and contact hypersensitivity in atopic dermatitis and rosacea. Exp Dermatol. 2013. 22:752-753.

Di Giacinto C, Marinaro M, Sanchez M, Strober W, Boirivant M. Probiotics ameliorate recurrent Th1-mediated murine colitis by inducing IL-10 and IL-10-dependent TGF- $\beta$-bearing regulatory cells. J Immunol. 2005. 174:3237-3246.

Grewe M, Bruijnzeel-Koomen CA, Schöpf E, Thepen T, LangeveldWildschut AG, Ruzicka T, et al. A role for Th1 and Th2 cells in the immunopathogenesis of atopic dermatitis. Immunol Today. 1998. 19:359-361.

Homey B, Steinhoff M, Ruzicka T, Leung DY. Cytokines and chem- okines orchestrate atopic skin inflammation. J Allergy Clin Immunol. 2006. 118:178-189.

Iida R, Saito K, Yamada K, Basile AS, Sekikawa K, Takemura M, et al. Suppression of neurocognitive damage in LP-BM5-infected mice with a targeted deletion of the TNF- $\alpha$ gene. FASEB J. 2000. 14:1023-1031.

Kalliomäki M, Isolauri E. Role of intestinal flora in the development of allergy. Curr Opin Allergy Clin Immunol. 2003. 3:1520.

Matsuda H, Watanabe N, Geba GP, Sperl J, Tsudzuki M, Hiroi J, et al. Development of atopic dermatitis-like skin lesion with IgE hyperproduction in NC/Nga mice. Int Immunol. 1997. 9:461466.

Murosaki S, Yamamoto Y, Ito K, Inokuchi T, Kusaka H, Ikeda H, et al. Heat-killed Lactobacillus plantarum L-137 suppresses naturally fed antigen-specific IgE production by stimulation of IL12 production in mice. J Allergy Clin Immunol. 1998. 102:5764.

Pochard P, Gosset P, Grangette C, Andre C, Tonnel AB, Pestel J, et al. Lactic acid bacteria inhibit $\mathrm{TH} 2$ cytokine production by mononuclear cells from allergic patients. J Allergy Clin Immunol. 2002. 110:617-623.

Shida K, Makino K, Morishita A, Takamizawa K, Hachimura S, Ametani A, et al. Lactobacillus casei inhibits antigen-induced IgE secretion through regulation of cytokine production in murine splenocyte cultures. Int Arch Allergy Immunol. 1998. 115:278287.

Strachan DP. Hay fever, hygiene, and household size. BMJ. 1989. 299:1259-1260.

Suto H, Matsuda H, Mitsuishi K, Hira K, Uchida T, Unno T, et al. NC/Nga mice: a mouse model for atopic dermatitis. Int Arch Allergy Immunol. 1999. 120:70-75.

van der Aa LM, Chadzinska M, Golbach LA, Ribeiro CMS, Verburg-van Kemenade BML. Pro-inflammatory functions of carp CXCL8-like and CXCb chemokines. Dev Comp Immunol. 2012. 36:741-750.

Werfel T, Wittmann M. Regulatory role of T lymphocytes in atopic dermatitis. Chem Immunol Allergy. 2008. 94:101-111.

Wollowski I, Rechkemmer G, Pool-Zobel BL. Protective role of probiotics and prebiotics in colon cancer. Am J Clin Nutr. 2001. 73:451S-455S.

Yazdanbakhsh M, Kremsner PG, van Ree R. Allergy, parasites, and the hygiene hypothesis. Science. 2002. 296:490-494.

Zhang M, Lin Y, Iyer DV, Gong J, Abrams JS, Barnes PF. T-cell cytokine responses in human infection with Mycobacterium tuberculosis. Infect Immun. 1995. 63:3231-3234. 